\title{
INFECTIVE ENDOCARDITIS IN CHILDREN: Experience of a Moroccan center
}

\author{
F. Bennaoui ${ }^{1,2}$, N. El IdrissiSlitine ${ }^{1,2}$, G. Draiss ${ }^{3}$, N. Rada ${ }^{3}$, M. Bouskraouiं, F.M.R Maoulainine ${ }^{1,2}$
}

\begin{abstract}
1: Neonatal Intensive Care Unit ,Mother and Child Hospital, Mohammed VI Marrakesh University Hospital, Morocco. 2:Childhood Health and Development Research Team, Marrakesh Medical School, Cadi Ayyad University, Morocco. 3: Pediatric Department A, Mother and Child Hospital, Mohammed VI Marrakesh University Hospital, Morocco.
\end{abstract}

\section{INTRODUCTION}

- Pediatric infective endocarditis (IE) is a potentially severe and lethal disease .

OBJECTIVES:

- Assess hospital incidence and management of IE

- Study the clinical, paraclinical and therapeutic profile of IE

- Study the outcome of this affection among children from south of Morocco

\section{MATERIAL AND METHODS}

- A retrospective study of cases of infective endocarditis

- At the Pediatric Department.

- Mohammed VI university hospital from January 2011 to October 2016.

- The inclusion criteria : cases diagnosed with IE of our series were selected according to the modified Duke criteria.

- We studied the epidemiological, clinical, microbiological data and complications of this disease.

RESULTS

- Our study found 10 cases in five years, which is $0.13 \%$ of admissions.

- The means age was 5.1 years (Extremes [1month, 15 years]), with a male predominance $(90 \%)$.

- 7 endocarditis were certain (70\%) and 3 endocarditis were possible $(30 \%)$

- Clinical presentation: infectious syndrome in all cases $(100 \%)$

- The inflammatory status was positive in $90 \%$ of cases.

- Streptococcus and staphylococcus were found in 6 cases $(60 \%)$.

- Blood cultures were negative in three cases $(30 \%)$

- The heart diseases were dominated by congenital ones $(80 \%)$, of which $50 \%$ were ventricular septal defect

- The treatment with bi antibiotics was the rule, for 7 children were treated by: cephalosporin 3rdgeneration + aminoglycoside, in 2 cases: the third antibiotic was added amoxicillin and in 1 case the third added antibiotic was : rifampicin-teicoplanin

- In 8 cases $(80 \%)$, there were good outcomes, 2 cases: neurological and thromboembolic complications caused by multiple cerebral abscesses and lower limb thrombophlebitis.

\section{DISCUSSION}

- Infectious endocarditis is rare among European children. The experiment of Lyon totals two to eight cases per year, without variations for 40 years [1].

- In Morocco, data on child endocarditis are poor. In our study, the prevalence found was $0.13 \%$ of admissions.

-This frequency is much lower than what we found in the European literature [2]

- Male predominance is frequently found in the literature.

\section{Congenital heart diseases $\quad$ n: $8(80 \%)$}

Ventricular Septal Defect

n:5 (50\%)

Auricular Septal Defect

n: 2 (20\%)

Others n:1 (10\%)

Positive blood cultures n:7 (70\%)

\begin{tabular}{cc} 
Complications & $\mathrm{n}: 2 \quad(20 \%)$ \\
Death & $\mathrm{n}: 1 \quad(10 \%)$ \\
\hline
\end{tabular}

Table I : Characteristics of the main results

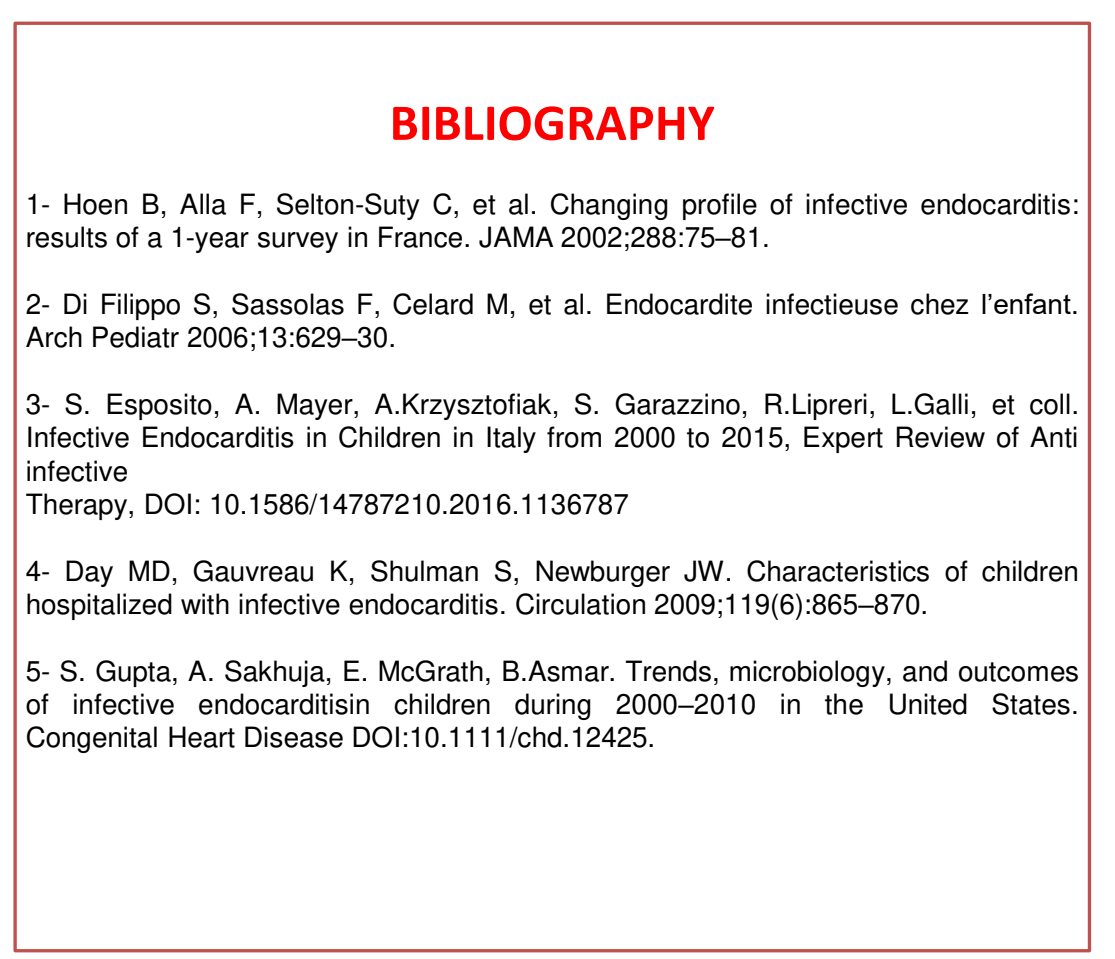

- Streptococcus and staphylococcus are the most frequently germs with more than $90 \%$ of positive blood cultures [3].

- Negative blood cultures are either explained by anterior antibiotherapy or caused by pathogens that are slowly growing or difficult to be found by standard techniques, however, negative blood culture does not rule out the diagnosis of infective endocarditis [3].

- The underlying heart diseases were dominated by congenital ones in this study. This could be explained by the admission modalities and the rarity of rheumatic valvulopathy; after the strategy of fighting rheumatic fever in Morocco

- The treatment is based on a probabilistic antibiotic and then adapted to the antibiogram, synergistic, bactericidal, parenteral and sufficiently long for 6 weeks. In our study, it lasted in average four weeks.

- Complications during endocarditis are cardiac and extracardiac. In several series, congestive heart failure was observed in 10 to $30 \%$ of patients [4].

- The overall mortality for IE cases in a US study was $2.8 \%$ [5], in this study, one child having pulmonary vegetation died from pulmonary embolism.

\section{CONCLUSION}

- IE occurs mostly in children with congenital heart disease, which may sometimes be the mode of revelation.

- The progression of IE can be characterized by complications, which are frequent and severe.

- Early diagnosis is valuable for adequate management.

- One child died from pulmonary embolism. 